\title{
Challenges associated and approaches for successful translation of nanomedicines into commercial products
}

\author{
"The successful clinical translation of nanomedicine has \\ challenges of reproducible manufacturing and scale-up, lack \\ of acceptable screening platforms and reliable techniques to \\ evaluate the efficacy, toxicity and stability in vivo."
}

First draft submitted: 27 January 2016; Accepted for publication: 13 February 2017; Published online: 24 March 2017

Keywords: characterization • clinical challenges $\bullet$ commercialization $\bullet$ manufacturing scale-up $\bullet$ nanomedicine $\bullet$ nanopharmaceuticals $\bullet$ regulatory challenges $\bullet$ reproducibility

Nanomedicines are complex engineered nanoscale structures and are widely used in the treatment and targeting of diseases because of their numerous therapeutic benefits. These benefits are: protection of biomolecules from degradation, enhanced solubility and bioavailability, improved pharmacokinetics, reduced toxicity, enhanced therapeutic efficacy, decreased drug's immunogenicity, delivery at the target site and diagnostics/ treatment options in one system [1-4]. Several nanomedicine products have been approved by the US Food and Drug Administration (FDA) and the European Medicines Agency (EMA) for various therapeutic indications $[2,5-6]$. High therapeutic efficacy and safety are the two primary qualifications for a product to become commercially successful. But, most of the nanomedicine products fail to accomplish these requirements and thus their successful commercialization [7]. Limited clinical success of nanomedicines is mainly due to challenges in formulation optimization, batch-to-batch consistency, robust and reproducible manufacturing and scale-up, characterization and screening methods, regulatory barriers and instability under biological environments [2,7-8]. Specific properties of nanomedicines those are responsible for their numerous benefits also generate safety and translational challenges.
Thus, understanding the fundamental, clinical and regulatory aspects of nanomedicine is of paramount significance. In this editorial, challenges associated with the successful translation of nanomedicines from preclinical to commercial stage and suggested approaches to achieve this translation are discussed. Nanopharmaceutical drug delivery and therapeutic systems are collectively referred as nanomedicines in this article.

\section{Challenges in manufacturing scale-} up \& reproducibility

A successful manufacturing and scale-up of nanomedicines under regulated conditions present unique challenges [5,7-9]. The nanoformulation process often involves multiple steps such as homogenization, sonication, centrifugation, solvent evaporation, extrusion, size reduction, lyophilization, sterilization, etc. At small-scale, the formulation/ process optimization and reproducibility can be achieved relatively easily; however, at large scale it poses a challenge to achieve a robust manufacturing process and minimize batchto-batch variability. This severely limits the clinical and/or commercial translation of nanomedicine [8]. The subtle variations in the formulation and/or manufacturing process can significantly affect the critical physicochemical properties such as size, crystallinity,
Vivek Agrahari

Author for correspondence:

Technical Development, Bayer U.S. LLC, Shawnee, KS 66210, USA

Tel.: +1 8168058048

vivek.agrahari@bayer.com

\section{Praveen Hiremath}

Formulation Technology, Bayer Animal Health GmbH, Leverkusen 51368, Germany 
drug-loading/-release profile, surface charge, chemistry, ultimately influencing the therapeutic outcomes. Conventional pharmaceutical industrial facilities are usually not well equipped for the manufacturing of nanomedicine products due to constraints in using organic solvents, capability to handle and process nanosized drugs/excipients due to environmental/operator/product safety concerns and requirement of sterile-manufacturing capability $[7,8]$. The physicochemical properties and integrity of nanomedicines also need to be maintained throughout the manufacturing process and during the shelf-life. In general, most industries have a well-established infrastructure to manufacture conventional solid, liquid, semisolid dosage forms, etc. However, nanomedicines manufacturing generally requires complicated and expensive approaches such as high-energy milling, high-pressure homogenization, lyophilization, etc. This demands in-depth process understanding, precise controls/checks, validated equipment/process train along with experienced staff to meet the critical quality attributes of the product such as size distribution, surface characteristics, charge, porosity and physicochemical stability $[5,8]$.

\section{Analytical characterization \& quality control challenges}

Lack of adequate characterization (in-process and quality control [QC]) methods and complexities involved in validating such methods poses a significant challenge to both the developers and regulators. The characterization of nanomedicines includes size, size distribution, morphology, surface charge, surface functionality, particle interactions/behavior, porosity, drug loading, solubility, $\mathrm{pH}$, viscosity, toxicity and stability analyses [5]. Therefore, the characterization of nanomedicines involves advanced approaches such as dynamic light scattering, electron microscopy, and fluorescence spectroscopy, etc., whereas most of the industries are usually equipped with standard analytical equipment. Techniques to analyze nanomedicines are not only expensive, but, also require a team of experts to perform the analysis and data interpretation. This substantially adds to the cost of the nanomedicines manufacturing and testing. Another major challenge is to find an appropriate sterilization method without compromising physicochemical properties of the nanomedicines critical for their performance. The lack of reliable techniques to analyze nanomedicines stability in vivo and under GMP environment is another major hurdle in their successful clinical translation [10].

\section{Pharmacological \& safety challenges}

Clinical translation of nanomedicine is suffering from the lack of acceptable and/or reliable screening plat- forms that can evaluate the efficacy and toxicity with a good in vivo correlation [5,7]. This is particularly more relevant in case of preclinical screening techniques and models of a meaningful relevance to human clinical data. The subtle changes in nanomedicines physicochemical properties arising from small deviations in the manufacturing process may result into altered pharmacokinetics, in vivo clearance, drug release kinetics and toxicity/safety profiling. The extremely small size of nanomedicines can profoundly influence the drug Adsorption, Distribution, Metabolism and Excretion (ADME). In vitro and in vivo evaluations characterize the product interactions with the biological system, however, the data obtained from current toxicity tests in preclinical models are not always extrapolatable to human. In vitro characterizations including drug release, cellular uptake, toxicity and immunology are mostly carried out using cells culture in monolayers (2D). However, nanoformulations uptake into cells and tissues is influenced by interactions among its own physicochemical characteristics. Therefore, 3D cell systems may provide better outcomes [11,12].

At present, preclinical testing for small-molecule drugs is considered adequate to assess the in vivo efficacy, toxicity, and ADME of nanomedicines, if performed in at least two animal models with multiple doses over an extended period of treatment [5]. However, preclinical data, especially immunotoxicity, cannot accurately predict the safety of nanomedicines in human $[5,8]$. Another issue with nanomedicines is environmental safety during the manufacturing process. The handling of a nanosized powder demands special caution since such particles are capable of skin penetration (dermal exposure) and may also lead to pulmonary toxicities $[8,13]$.

\section{Regulatory challenges}

The lack of specific regulatory guidelines for preclinical development and characterization of nanomedicine products at biophysiological level has hampered their potential in clinical practice [5,7]. Hurdles in regulatory pathways for nanomedicines translation are primarily due to the complexity of the product and the evaluation processes of the small-molecule drugs are frequently adapted [6]. Hence, there is a critical need to develop a comprehensive list of tests that cover the characterization, pharmacology, and toxicology of nanomedicines. Also, a fundamental question for nanomedicine translation is if the product meets the criteria of a scientifically acceptable definition of what can be considered a nanomedicine. Currently, when considering whether an FDA-regulated product involves the application of nanotechnology, the FDA parameters are: Whether a material or end product is 
engineered to have at least one external dimension, or an internal or surface structure, in the nanoscale range $(\sim 1-100 \mathrm{~nm})$; and Whether a material or end product is engineered to exhibit properties or phenomena, including physical or chemical properties or biological effects that are attributable to its dimension(s), even if these dimensions fall outside the nanoscale range, up to $1000 \mathrm{~nm}$ [14]. Since the nanomedicine field is rapidly evolving and challenging, the FDA and EMA, along with other medical regulatory agencies suggest that the evaluation and approval of nanomedicine products are performed on a case-by-case basis. Therefore, the optimal approach used will be flexible, iterative, scientific, collaborative and product-focused to facilitate innovation [15].

\section{Conclusion \& opinion on nanomedicines' successful translation}

The application of nanomedicines in healthcare is changing the current diagnosis and therapy concepts. Indeed, despite the significant impact of nanomedicines in healthcare, only a few products have reached the market. Thus, a better understanding of critical physicochemical characteristics as well as in vivo behavior of nanoformulations is needed. Implementation of a robust QC system and incorporating quality-by-design approaches can identify critical quality parameters to ensure batch-to-batch reproducibility, high-throughput optimization and the process/formulation variables for a better product profile [16]. Integration of development steps into one system may also bring exciting advances in the nanomedicines clinical translation and an opportunity for future research to significantly reduce the time and cost. Furthermore, advanced technologies should be developed to formulate new generation nanomedicines with desired physicochemical characteristics. This can be made through combinatorial approaches of microfluidics and other automation techniques as a screening tool to identify an optimal nanomedicine formulation [11,17,18].

Successful translation of nanomedicines implies upgrading of industrial infrastructures. This includes setting up equipped characterization lab, good manufacturing and pilot manufacturing/scale-up facilities according to regulatory standards. It is essential that nanomedicine manufacturing includes QC checks at every step to maintain the quality and efficacy of the batches [8]. Thus, sensitive and robust analytical techniques that are able to detect minute variations and adequately characterize the product should be established. Also, using multiple methods complementing each other to evaluate the same parameter is time saving. This necessitates the collaboration between $\mathrm{R} \& \mathrm{D}$, QC and manufacturing subdivisions. It is also impor- tant that the traditional lab-scale manufacturing process of a nanomedicine product is industrially viable. The environmental safety issue with nanomanufacturing can be solved by adopting the processing within an environment that has considerably lower safety concern such as an aqueous environment.

\section{"To bridge the gap of nanomedicine lab research to industrial manufacturing, collaboration and integration among academicians, scientists, industries, and regulatory agencies is required to develop comprehensive approaches to ensure safe, effective, and translatable nanomedicine products."}

The lack of adequate criteria and validated techniques to characterize nanomedicines toxicity is also a major concern [5,7-8]. Most toxicology assays are shortterm, and it is therefore essential to evaluate whether these tests are predictive in providing long-term toxicity. Hence, an in vitro and in vivo characterization cascade of safety and efficacy testing is needed to accelerate the translation of nanomedicine [19,20]. Nanomedicines characterization is often performed under conditions that do not exactly reflect the real physiological and biological environments. Therefore, incorporating novel methods such as microfluidics are promising options for creating environments that mimic in vivo conditions [21]. Furthermore, selection of appropriate animal models is important [22] and the differences in sensitivity/specificity to the given nanoformulation should be considered.

The application of nanotechnology in medicine has led to significant progress in providing better health outcomes. According to Grand View Research, Inc., the global nanomedicine market is likely to reach $\$ 344.0$ billion by 2024 . New innovations in healthcare are likely to bring more nanomedicine products to the market. Therefore, it is important to examine whether the methods and regulatory guidelines are able to adequately assess the delivery, fate, safety and efficacy of nanomedicines. Attracting investment for nanomedicine research is particularly challenging. It is therefore essential to demonstrate the social-/economic-added values such as cost-effectiveness of nanomedicine products compared with established treatments before commercialization. A significant commercial challenge also involves the physicians and patients acceptance of nanomedicine products. Approaches that could significantly enhance the nanomedicines translation may include:

- Use of science-based quality by design approaches in the formulation development, robust manufacturing, and scale up to ensure batch-to-batch 
reproducibility and product quality at commercial scale;

- Profound knowledge of the key physicochemical attributes with the adequate characterization and control;

- Meaningful in vitro and ex vivo models relevant to the specific routes of administration and with in vivo relevance/significance;

- Understanding of the ADME and its relationship to product characteristics to predict the in vivo effects;

- Enhanced understanding of nanomedicines interactions with the biological system;

- Development of standardized and validated assays for analyzing stability, safety and efficacy;

- Continued research, strong collaboration and indepth interactions with academia, industry and

\section{References}

1 Peer D, Karp JM, Hong S, Farokhzad OC, Margalit R, Langer R. Nanocarriers as an emerging platform for cancer therapy. Nat. Nanotechnol. 2(12), 751-760 (2007).

2 Anselmo AC, Mitragotri S. Nanoparticles in the clinic. Bioeng. Transl. Med. 1(1), 10-29 (2016).

3 Agrahari V, Agrahari V, Mitra AK. Nanocarrier fabrication and macromolecule drug delivery: challenges and opportunities. Ther. Deliv. 7(4), 257-278 (2016).

4 Meng J, Agrahari V, Youm I. Advances in targeted drug delivery approaches for the central nervous system tumors: the inspiration of nanobiotechnology. J. Neuroimmune. Pharmacol. 12(1), 84-98 (2016).

5 Ragelle H, Danhier F, Preat V, Langer R, Anderson DG. Nanoparticle-based drug delivery systems: a commercial and regulatory outlook as the field matures. Expert. Opin. Drug. Deliv. doi:10.1080/17425247.2016.1244187 (2016) (Epub ahead of print).

6 Sainz V, Conniot J, Matos AI et al. Regulatory aspects on nanomedicines. Biochem. Biophys. Res. Commun. 468(3), 504-510 (2015).

7 Kaur IP, Kakkar V, Deol PK, Yadav M, Singh M, Sharma I. Issues and concerns in nanotech product development and its commercialization. J. Control. Release 193, 51-62 (2014).

8 Desai N. Challenges in development of nanoparticle-based therapeutics. AAPS J. 14(2), 282-295 (2012).

9 Muthu MS, Wilson B. Challenges posed by the scale-up of nanomedicines. Nanomedicine (Lond.) 7(3), 307-309 (2012). regulatory organizations to further understand and develop nanomedicines regulatory and safety guidelines.

Overall, to bridge the gap of nanomedicines lab research to industrial manufacturing, collaboration and integration among academicians, scientists, industries, and regulatory agencies are required in a harmonized manner to develop comprehensive approaches to ensure safe, effective and translatable nanomedicine products.

Financial \& competing interests disclosure

The authors have no relevant affiliations or financial involvement in any organization or entity with a financial interest in or financial conflict with the subject matter or materials discussed in the manuscript. This includes employment, consultancies, honoraria, stock ownership or options, expert testimony, grants or patents received or pending, or royalties.

No writing assistance was utilized in the production of this manuscript.

10 Landesman-Milo D, Peer D. Transforming nanomedicines from lab scale production to novel clinical modality. Bioconjug. Chem. 27(4), 855-862 (2016).

11 Bhise NS, Ribas J, Manoharan V et al. Organ-on-a-chip platforms for studying drug delivery systems. J. Control. Release 190, 82-93 (2014).

12 Gupta N, Liu JR, Patel B, Solomon DE, Vaidya B, Gupta V. Microfluidics-based 3D cell culture models: utility in novel drug discovery and delivery research. Bioeng. Transl. Med. 1(1), 63-81 (2016).

13 Nel A, Xia T, Madler L, Li N. Toxic potential of materials at the nanolevel. Science 311(5761), 622-627 (2006).

14 US FDA. Considering Whether an FDA-Regulated Product Involves the Application of Nanotechnology. http://www.fda.gov

15 Hamburg MA. Science and regulation. FDA's approach to regulation of products of nanotechnology. Science 336(6079), 299-300 (2012).

16 Troiano G, Nolan J, Parsons D, Van Geen Hoven C, Zale $\mathrm{S}$. A quality by design approach to developing and manufacturing polymeric nanoparticle drug products. AAPS J. 18(6), 1354-1365 (2016).

17 Bjornmalm M, Yan Y, Caruso F. Engineering and evaluating drug delivery particles in microfluidic devices. J. Control. Release 190, 139-149 (2014).

18 Valencia PM, Farokhzad OC, Karnik R, Langer R. Microfluidic technologies for accelerating the clinical translation of nanoparticles. Nat. Nanotechnol. 7(10), 623-629 (2012).

19 Dobrovolskaia MA. Pre-clinical immunotoxicity studies of nanotechnology-formulated drugs: challenges, considerations and strategy. J. Control. Release 220(Pt B), 571-583 (2015). 
20 Jones CF, Grainger DW. In vitro assessments of nanomaterial toxicity. Adv. Drug. Deliv. Rev. 61(6), 438-456 (2009).

21 Lim JM, Karnik R. Optimizing the discovery and clinical translation of nanoparticles: could microfluidics hold the key? Nanomedicine (Lond.) 9(8),

1113-1116 (2014).

22 Jang HL, Zhang YS, Khademhosseini A. Boosting clinical translation of nanomedicine. Nanomedicine (Lond.) 11(12), 1495-1497 (2016) 